\title{
Diagnostic and prognostic value of biomarkers in acute myocardial infarction
}

\author{
Yuqi Chen, Yifei Tao, Lan Zhang, Weiting Xu, Xiang Zhou ${ }^{\odot}$
}

Department of Cardiology, The Second Affiliated Hospital of Soochow University, Suzhou, China

\section{Correspondence to} Xiang Zhou and Weiting $\mathrm{Xu}$, Department of Cardiology, The Second Affiliated Hospital of Soochow University, Suzhou, China;

zhou-xiang@suda.edu.cn, xuwt1968@aliyun.com

YC and YT contributed equally.

Received 14 January 2019 Revised 25 February 2019 Accepted 26 February 2019 Published Online First 4 April 2019

\section{Check for updates}

(c) Author(s) (or their employer(s)) 2019. No commercial re-use. See rights and permissions. Published by BMJ.

To cite: Chen Y, Tao Y, Zhang L, et al. Postgrad Med J 2019:95:210-216

\section{ABSTRACT}

The incidence of acute myocardial infarction (AMI) has been increasing rapidly in recent years, seriously endangering human health. Cardiac biomarkers play critical roles in the diagnosis and prognosis of AMI. Troponin is a highly sensitive and specific biomarker for AMI diagnosis and can independently predict adverse cardiac events. Other biomarkers such as $\mathrm{N}$-terminal $\mathrm{B}$-type natriuretic peptide and $\mathrm{C}$ reactive protein are also valuable predictors of cardiovascular prognosis. Recently, several novel biomarkers have been identified for the diagnosis and risk assessment in patients with AMI. A multibiomarker approach can potentially enhance the diagnostic accuracy and provide more information for the early risk stratification of AMI. In this review, we will summarise the biomarkers discovered in recent years and focus on their diagnostic and prognostic value for patients with AMI.

\section{INTRODUCTION}

Biomarkers of cardiac injury have been used to diagnose acute myocardial infarction (AMI) for over half a century. Karmen et $a l^{1}$ first reported the elevation of aspartate transaminase (AST) in patients with AMI in 1954. AST was the first cardiac biomarker used in clinical practice. However, it became clear that AST had limited use as a cardiac biomarker due to the lack of specificity for myocardial injury. ${ }^{23}$

In the following years, creatine kinase (CK) was defined as a more sensitive and specific biomarker and lactate dehydrogenase (LDH) was also discovered. However, specificity continued to be problematic, especially for patients with muscle and liver disease or injury. Later development of new detection methods identified CK-MB as a molecule showing higher diagnostic accuracy. Unfortunately, $\mathrm{CK}, \mathrm{LDH}$ and CK-MB still lacked specificity for diagnosing AMI. ${ }^{45}$

Rapid advances in laboratory techniques and the clinically urgent need resulted in the discovery of cardiac troponins (cTns). ${ }^{6}$ The guidelines have recommended cTn as the preferred biomarker due to its sensitivity and cardiac specificity. ${ }^{7}$ In this review, we summarise the biomarkers discovered in recent years and focus on their diagnostic and prognostic value in AMI.

\section{SUMMARY OF CARDIAC BIOMARKERS Myocardial necrosis biomarkers Cardiac troponins (cTns)}

Troponins are important for actin and myosin interaction, and regulation of cytoplasmic contractile function in response to cytosolic calcium and protein phosphorylation. The troponin complex is located along with tropomyosin on the actin filament. Cardiac-specific isoforms of cTnI and cTnT exist in myocardial tissue, whereas troponin $\mathrm{C}$ is also expressed in skeletal muscle, rendering it unsuitable for the purposes of AMI diagnostic. ${ }^{8}$

The Third Universal Definition of Myocardial Infarction requires detection of a significant elevation and decline in serum cTn level with at least one value above the 99th percentile of the upper reference limit; moreover, cTn levels should be measured with a coefficient of variation of $\leq 10 \%$. ${ }^{9}$ The development of high-sensitivity troponin (hs-cTn) analysis improved the identification of myocardial injury, with abnormal cTn detected within 24 hours. ${ }^{10-13}$

Although elevated serum cTn levels reflect myocardial damage, the mechanistic basis for this observation remains unclear. In addition to spontaneous AMI following acute coronary occlusion and plaque rupture, AMI can be secondary to ischaemia resulting from increased demand for or decreased supply of oxygen, coronary embolism, coronary artery spasm, arrhythmia and hypertension. Consequently, cTn levels may be upregulated in coronary disease and also in non-cardiac disorder. ${ }^{14}{ }^{15}$ Indeed, cTn level is increased in patients with renal failure without symptoms of acute coronary syndrome (ACS), although they are at increased risk of cardiac abnormalities. Another study reported that $50 \%$ of patients with renal failure and high cTn level had coronary arteries free of flow-limiting stenosis in an angiography, ${ }^{16}$ implying that cTn is chronically elevated in these individuals.

High cTn level is useful for diagnostic purpose and is also an independent prognostic marker, as evidenced in several clinical trials and a meta-analysis. $^{17}$ cTn level can inform clinical decision-making in terms of whether a more aggressive or conservative treatment course should be adopted after ACS since abnormalities can identify subgroups of patients greater benefit from early invasive therapy. ${ }^{19}$

\section{Myoglobin}

Myoglobin is a low molecular weight cytoplasmic heme protein that is the most sensitive conventionally assayed biomarker of AMI. Nevertheless, myoglobin has lower specificity for cardiac necrosis than cTnI and its expression may be upregulated in non-cardiac disorders, such as skeletal muscle disease or injury and chronic renal disease. ${ }^{20} 21$ Despite lack of cardiac specificity, combining myoglobin with troponin significantly improved the ability to identify those at risk of 
increased mortality from AMI as compared with either marker individually. ${ }^{22}{ }^{23}$ Myoglobin is primarily eliminated through the kidneys, and renal insufficiency is recognised as a predictor of adverse outcome, including mortality, in patients with AMI. ${ }^{24}$ Thus, it is presumed that myoglobin predicts mortality by identifying patients with renal insufficiency.

\section{Ischaemia-modified albumin}

In acute ischaemia, the $\mathrm{N}$ terminus of albumin is altered, thereby reducing its binding capacity, and the resultant protein is referred to as ischaemia-modified albumin (IMA). In patients with suspected ACS, the diagnostic accuracy at presentation increased when IMA was used in conjunction with cTnT and ECG findings. ${ }^{25}$ In fact, IMA in combination with initial cTnT is more sensitive than the latter alone for predicting adverse cardiac events, although the specificity and sensitivity for IMA are too low to be useful for clinical decision-making. ${ }^{26}$

\section{Heart-type fatty acid binding protein (hFABP)}

hFABP is a small cytoplasmic protein present in cardiomyocytes that is similar to myoglobin. It is rapidly released into the circulation after the onset of myocardial injury owing to its low molecular weight and cytoplasmic localisation. ${ }^{27}$ Agnello $e t a l^{28}$ found that early diagnosis of AMI was possibly based on measurement of hFABP level. However, hFABP expression is not cardiac specific, being detected at low levels in skeletal muscle and kidney. ${ }^{29}$ The diagnostic value of hFABP remains controversial. The prognostic value of hFABP in patients with suspected ACS has been investigated in several studies. High hFABP level was the best predictor of adverse events during a 1-year follow-up in patients with non-ST-elevation MI (NSTEMI) among markers of myocardial necrosis (hFABP, cTnI and CK-MB). ${ }^{30}$ Serial measurements of hFABP at the time of admission and discharge provided information about cardiac events in patients with chronic heart failure, and those with persistent elevation of hFABP had the highest rate of cardiac events, including cardiac deaths and rehospitalisations. ${ }^{31}$ However, hFABP has not been accepted as a standard biomarker of ACS, owing to certain technical issues that are discussed elsewhere. ${ }^{32}$

\section{Neuroendocrine biomarkers BNP/NT-proBNP}

B-Type natriuretic peptide (BNP) is a hormone secreted by cardiomyocytes in the heart ventricles in response to cardiac stress and ventricular dysfunction. After its synthesis the proBNP precursor is cleaved into the active BNP hormone and an inactive NT-proBNP fragment. The functions of BNP include vasodilatation, natriuresis and inhibition of the renin-angiotensin-aldosterone system. ${ }^{33}$

Serum levels of cardiac natriuretic peptides, especially BNP and NT-proBNP, are upregulated following ACS. ${ }^{34}$ Elevated BNP levels in patients with AMI were also found to be associated with myocardial infarct size. ${ }^{35}$ Although BNP/NT-proBNP levels are increased in patients with ACS, they cannot be used as diagnostic markers since they are also upregulated in other conditions with similar symptoms such as heart failure and pulmonary embolus. Due to partial clearance of BNP and NT-proBNP by renal excretion, patients with renal insufficiency also have high levels of BNP and NT-proBNP. ${ }^{36}$

Several studies have demonstrated the prognostic value of BNP and NT-proBNP in patients with myocardial infarction. Plasma NT-proBNP, measured 2 to 4 days after AMI, independently predicted left ventricular function and 1-year survival. ${ }^{37}$ Increased BNP level at initial presentation of patients with STEMI was associated with impaired reperfusion after fibrinolysis and higher 30-day mortality. After adjusting for cTnI, BNP remained independently associated with mortality and the rates of heart failure and death increased with higher baseline concentrations of BNP. ${ }^{38}$

It was reported that BNP and NT-proBNP were better at predicting cardiovascular events than Thrombolysis In Myocardial Infarction (TIMI) or Global Registry of Acute Coronary Events (GRACE) risk score. The predictive value of NT-proBNP was independent and the combination of NT-proBNP and TIMI or GRACE score did not significantly improve prediction of short-term mortality risk. ${ }^{39} 40$

Both BNP and NT-proBNP are excellent biomarkers of adverse events post-AMI; however, there is no consensus on whether they can be used to guide early treatment in order to improve patients' outcomes because of their demonstrated role in the diagnosis and management of heart failure.

\section{Adrenomedullin}

Adrenomedullin is a cardiovascular regulatory peptide whose levels are increased in the serum of patients with cardiovascular disease. It attenuates infarct development during acute myocardial injury and can potentially influence the pathological process in both the acute phase of AMI and subsequent remodelling. Elevated adrenomedullin levels are indicative of cardiac remodelling and may improve risk stratification in heart failure and MI. ${ }^{41} 42$

\section{Renin-angiotensin-aldosterone system (RASS)}

The RAAS is a hormone system that regulates blood pressure and fluid balance. It is activated following AMI, by the increased volume blood and vasoconstriction. Aldosterone promotes a broad range of detrimental cardiovascular effects during AMI, including acute endothelial dysfunction, increased endothelial oxidative stress, cardiac myocyte necrosis, and myocardial hypertrophy and fibrosis.

With the exception of BNP, the aforementioned neuroendocrine markers are not yet routinely used in clinical practice for diagnosis or prognosis. However, studies have shown that treatment of patients with AMI with inhibitors of the neuroendocrine system reduced morbidity and mortality, for instance, rates of death and heart failure in patients with ACS decreased by administration of ACE inhibitor/angiotensin receptor blocker and aldosterone inhibitors. ${ }^{43} 44$

\section{Inflammatory biomarkers}

\section{$C$ reactive protein (CRP)}

Inflammation plays an important role in the pathogenesis of atherothrombosis and ACS. CRP is an acute-phase inflammatory response biomarker, produced by hepatocytes on stimulation by inflammatory cytokines, primarily interleukin-6 (IL-6), that associated with increased cardiovascular risk in patients with established atherosclerosis. Several studies have reported that upregulation of CRP is an independent prognostic marker of recurrent non-fatal MI or cardiac death. ${ }^{45}{ }^{46}$ It also reflects the extent of myocardial injury in STEMIs. Wang $e a^{4 l^{47}}$ found that serum CRP was increased in patients with AMI suggesting that circulating CRP is a potential diagnostic biomarker. Despite some positive results, CRP is not considered to have sufficient specificity and sensitivity for use as a reliable diagnostic marker. ${ }^{4849}$

Nonetheless, CRP is a significant predictor of poor outcome. Lukin et $a l^{46}$ found that plasma CRP level predicted major 
Table 1 Summary of biomarkers in acute myocardial infarction

\begin{tabular}{|c|c|c|c|}
\hline Biomarkers & & Diagnostic value & Prognostic value \\
\hline \multirow[t]{6}{*}{ Myocardial necrosis } & $\mathrm{AST}^{1-3}$ & First biomarker of AMI but lacks specificity & No data \\
\hline & $\mathrm{CK} / \mathrm{CK}_{-} \mathrm{MB}^{4}$ & Not as sensitive or specific as cTn & Not consistently predictive of adverse events \\
\hline & Troponin $^{5-19}$ & Highly sensitive and specific, current 'gold standard' for AMI diagnosis & Independent predictor of adverse events \\
\hline & $\begin{array}{l}\text { Myoglobin } \\
20-24\end{array}$ & $\begin{array}{l}\text { Sensitive early after symptom onset, negative predictive value, but lacks } \\
\text { specificity }\end{array}$ & Predictive of mortality in renal insufficiency \\
\hline & $\mathrm{IMA}^{2526}$ & Less sensitive than cTn and lacks specificity & Possibly predictive of adverse events \\
\hline & hFABP ${ }^{27-32}$ & Sensitive early after symptom onset, but lacks specificity & Predictive of mortality \\
\hline \multirow[t]{3}{*}{ Neuroendocrine } & BNP/NT-proBNP $33-40$ & Not sensitive or specific & Highly predictive of heart failure and mortality \\
\hline & $\begin{array}{l}\text { Adrenomedullin } \\
4142\end{array}$ & & Possibly predictive of adverse events \\
\hline & RASS $^{43}$ & & \\
\hline \multirow[t]{8}{*}{ Inflammation } & $\mathrm{CRP}^{47-54}$ & Not sensitive or specific. The diagnostic accuracy of cTn can be & Possibly predictive of heart failure and mortality \\
\hline & IL- $6^{55-57}$ & increased when combining with some of these inflammatory & \\
\hline & TNF- $\alpha^{58}$ & & \\
\hline & $\mathrm{PCT}^{59-61}$ & & \\
\hline & $\mathrm{MPO}^{62-64}$ & & \\
\hline & $M_{M} 6566$ & & \\
\hline & $\mathrm{SCD} 40 \mathrm{~L}^{67}$ & & \\
\hline & $\mathrm{PGF}^{68}$ & & \\
\hline \multirow{4}{*}{$\begin{array}{l}\text { Other novel cardiac } \\
\text { biomarkers }\end{array}$} & miRNAs ${ }^{69-78}$ & Raised but not sensitive or specific for AMI & Possibly predictive of mortality and heart failure \\
\hline & $\mathrm{ST} 2^{79-81}$ & Raised but not sensitive or specific for AMI & Predictive of mortality and heart failure \\
\hline & GDF-15 $5^{82-84}$ & Raised but not sensitive or specific for AMI & Predictive of mortality and ischaemia \\
\hline & Gal-3 $3^{85-89}$ & Raised in AMI but insufficient data & Possibly predictive of adverse events \\
\hline
\end{tabular}

AMI, acute myocardial infarction; cTn, cardiac troponin; IMA, ischaemia-modified albumin.

adverse cardiac events in patients with ACS, and other studies confirmed that a higher CRP level is associated with increased long-term risk of recurrent cardiovascular events or death. ${ }^{50}$ Serum concentration of high-sensitivity CRP (hs-CRP) is a sensitive indicator of inflammation which is closely related to plaque formation and is an independent prognostic marker in patients with $\mathrm{ACS}^{5152}$; it has therefore been recommended as a guide for treatment decisions. However, several studies showed that CRP is unrelated to the occurrence of AMI, and that only cTn, not CRP, is useful for identifying patients who will benefit from an invasive strategy or antithrombotic treatment. ${ }^{53} 54$

\section{Interleukin-6 (IL-6) and tumor necrosis factor- $\alpha$ (TNF- $\alpha$ )}

IL- 6 and TNF- $\alpha$ are two major pro-inflammatory markers. The former is involved in inflammatory cell activation and recruitment, and stimulates the liver to produce acute-phase proteins such as CRP while exerting a negative inotropic effect through myocardial nitric oxide synthase (NOS). Wang et $a l^{55}$ found that IL- 6 was related to the procession of ACS and had prognostic use. Serum IL-6 was shown to be useful for predicting mortality in patients with $\mathrm{ACS}^{51}$ and identifying individuals who could benefit from targeted interventional or intensive therapy. ${ }^{56}$ However, the circadian fluctuation in IL-6 levels and a lack of confirmatory studies have limited the use of IL- 6 as an effective biomarker of ACS. ${ }^{57}$ TNF- $\alpha$ is a cardio-inhibitory cytokine, detected in several tissues including endothelial cells, smooth muscle cells and macrophages that reduces cardiac contractility either directly or via induction of NOS. It was reported that inflammatory cytokines, including TNF- $\alpha$ and hs-CRP, could predict the 6-month survival of patients with ACS. ${ }^{58}$

\section{Procalcitonin}

Procalcitonin (PCT) is a peptide precursor of the hormone calcitonin, which is involved in calcium homeostasis. It has also been shown to be induced under inflammatory conditions. Kafkas et $a l^{59}$ showed that the PCT concentration was increased in patients with AMI. Other studies also have found that PCT have prognostic value for predicting mortality or other ischaemic events. ${ }^{6061^{* * * *}}$

\section{Markers of plaque destabilisation}

Myeloperoxidase (MPO) is a component of granules within the neutrophils. It plays a crucial role in inflammation and oxidative stress in the cellular level. ${ }^{62}$ Studies have shown that MPO is inferior to current biomarkers for diagnostic purposes, but elevated levels independently predict future risk of coronary artery disease in both patients with ACS and healthy individuals. ${ }^{63}$ Among patients with ACS, baseline MPO has been shown to be an independent predictor of adverse cardiac events. Omran et $a l^{64}$ also found that the use of baseline levels of three biomarkers in combination (MPO, CK-MB and cTnI) could increase early diagnosis accuracy of AMI.

Matrix metalloproteinases (MMPs) are a family of zinc-containing endoproteinases, which plays an important role in the physiology and pathology of the cardiovascular system. ${ }^{65} \mathrm{MMPs}$, in particular MMP-9, are important in the collagen breakdown and structural changes associated with ventricular remodelling after AMI and a distal biomarker for inflammation. ${ }^{66}$

\section{Markers of myocyte rupture}

The CD40 and CD40 ligand (CD40L) system is expressed on a variety of cell types. Studies suggest that elevated serum levels of soluble CD40L (sCD40L) identify patients with ACS at higher risk of recurrent $\mathrm{MI}$ and death independent of other prognostic biomarkers including cTnI and CRP, ${ }^{67}$ and the subgroup of patients who will benefit from antiplatelet treatment can be identified. 
Placental growth factor (PGF) is associated with angiogenesis and vasculogenesis, which has been implicated in neovascularisation in ischaemic myocardium and promoting atherosclerosis. Higher concentration of PGF after ACS is associated with longterm risk of recurrent cardiovascular events independent of traditional risk factors. ${ }^{68}$

\section{Other novel cardiac biomarkers MicroRNAs (miRNAs)}

In recent years, several cardiac-specific miRNAs have been identified to play important roles in the development of cardiovascular diseases. ${ }^{69}$ Among these miRNAs, four cardiac-enriched miRNAs (miR-208, miR-499, miR-1 and miR-133) are consistently found to be increased in the plasma of patients with AMI. ${ }^{70}$ Devaux et $a l^{71}$ indicated that concentrations of miR-208b, miR-499 and miR-320a were significantly elevated in patients with AMI. Recently, miR-92a and miR-181a were used as potential biomarkers for the diagnosis of AMI. ${ }^{72} 73$ Oerlemans et $a l^{74}$ revealed that the combination of miR-1, miR-21 and miR-499 had a higher diagnostic value than hs-troponin $\mathrm{T}$. In addition to diagnostic value, miRNAs may be more useful for risk stratification and can be used as prognostic biomarkers. ${ }^{75}$ Circulating miRNA-197 and miRNA223 were identified as predictors for cardiovascular death in a large patient cohort with coronary artery disease. ${ }^{76}$ Studies also found that miRNA-134, miRNA-328, miRNA-34a and miRNA-208b were associated with the development of heart failure and increased risk of mortality after AMI. ${ }^{77} 78$

\section{Suppression of tumourigenicity 2 (ST2)}

ST2 is a biomarker of cardiomyocyte stress and fibrosis, which has been shown to be induced in conditions of MI and acute heart failure which causes myocardial overload. ${ }^{79}$ Studies have reported elevated level of ST2 in patients with ACS but would not be a reliable diagnostic marker because of the lack of specificity. These studies also suggested that ST2 has the ability to predict mortality and heart failure in patients with ACS. ${ }^{8081}$

\section{Growth differentiation factor-15 (GDF-15)}

GDFs are a protein subfamily belonging to the transforming growth factor beta superfamily. GDF-15 increases during tissue injury and inflammatory states and is associated with cardiometabolic risk. ${ }^{82}$ Studies have suggested that circulating concentration of GDF-15 is elevated in patients with $\mathrm{AMI}^{83}$ and can independently predict mortality or a composite of death and non-fatal AMI. Other studies also found that risk stratification can be improved by measuring both cTnT/ NT-proBNP and GDP-15 levels. ${ }^{84}$

\section{Galectin-3 (Gal-3)}

Gal-3 is involved in cardiac fibrosis, inflammation and reconstruction processes. Several studies have showed that circulating Gal-3 levels are elevated in AMI. ${ }^{85} 86$ In addition, Gal-3 is associated with ventricular remodelling and elevated plasma Gal-3 levels are related to lower left ventricular ejection fraction. ${ }^{87}$ Moreover, Gal-3 is also suggested as a valuable predictive marker in patients with coronary artery disease and ischaemic stroke. $^{88} 89$

\section{MULTIBIOMARKER APPROACH}

Ideal biomarkers should show high sensitivity, especially in the first few hours after symptom onset. ${ }^{90} \mathrm{~A}$ single cTn test may not provide sufficient sensitivity for early diagnosis of
AMI owing to its delayed release, even in the case of hs-cTn. Distinct pathways in the complex pathophysiology of AMI have been revealed by the identification of novel cardiac biomarkers. Several studies have suggested that adding tests for different biomarkers to cTn detection can improve diagnosis as well as prediction of short-term and long-term prognosis. Furthermore, the rational basis for cardiovascular risk assessment can be enhanced by employing a multibiomarker strategy that relates to various aspects of ACS.

Baseline levels of cTnI, CRP and BNP are independent predictors of adverse events following AMI. Kim et al $l^{91}$ confirmed that a combination of hs-CRP and NT-proBNP had greater value in predicting short-term cardiac events in patients with ACS than either marker alone. It was also demonstrated that combining biomarkers across the axes of myocardial stress, inflammation and myocyte necrosis improved prediction of cardiovascular death or heart failure. ${ }^{92}$ Additionally, low-risk patients identified based on negativity for $\mathrm{cTnI} / \mathrm{CK}-\mathrm{MB} /$ myoglobin had a low mortality. ${ }^{93}$ Other marker combinations include hFABP/cTn, which improved early diagnostic use ${ }^{94}$ compared with cTn only and hFABP/NT-proBNP/cTnT, ${ }^{95}$ IL-10/MPO/PGF/cTnT ${ }^{96}$ and

Main messages

The incidence of acute myocardial infarction (AMI) has been increasing rapidly in recent years.

- Cardiac biomarkers play critical roles in the diagnosis and prognosis of AMI.

- The multi-biomarker approach can increase the diagnostic accuracy and provide more information for the risk stratification of AMI.

Current research questions

Are there other novel biomarkers for the diagnosis of AMI in addition to troponin?

- Which biomarkers can be used to evaluate the prognosis of patients with AMI?

Key references

- Newby LK, Storrow AB, Gibler WB, et al. Bedside multimarker testing for risk stratification in chest pain units: the chest pain evaluation by creatine kinase-MB, myoglobin, and troponin I (CHECKMATE) study. Circulation 2001;103:1832-7.

- Richards AM, Nicholls MG, Espiner EA, et al. B-type natriuretic peptides and ejection fraction for prognosis after myocardial infarction. Circulation 2003;107:2786-92.

- Morrow DA, Cannon CP, Jesse RL, et al. National Academy of Clinical Biochemistry Laboratory Medicine Practice Guidelines: clinical characteristics and utilization of biochemical markers in acute coronary syndromes. Clin Chem 2007;53:552-74.

- Mueller C. Biomarkers and acute coronary syndromes: an update. Eur Heart J 2014;35:552-6.

- McCann CJ, Glover BM, Menown IB, et al. Prognostic value of a multimarker approach for patients presenting to hospital with acute chest pain. Am J Cardiol 2009;103:22-8. 
Self-assessment questions

1. Acute myocardial infarction is diagnosed based on the following evidence:

a. Typical chest pain lasts more than half an hour

b. Dynamic evolution of ECG

c. Cardiac dysfunction detected by echocardiography

d. Increased serum levels of troponin

2. Which biomarkers are sensitive at the early stage of acute myocardial infarction?
a. Myoglobin
b. AST
c. CKMB
d. Troponin

3. Which of the following biomarkers has the optimal sensitivity and specificity for diagnosing acute myocardial infarction?
a. Myoglobin
b. AST
c. CKMB
d. Troponin

4. Which biomarkers can be used to evaluate the prognosis of patients with myocardial infarction?
a. Troponins
b. hFABP
c. NT-proBNP
d. CRP

5. Which of the following descriptions is correct?
a. We should search for novel biomarkers
b. Serum biomarkers are golden standard for diagnosing diseases
c. We should develop multibiomarker approach
d. Current biomarkers are sufficient enough

ST2/GDF-15/hFABP/hs-TnT, ${ }^{97}$ which have been proposed as prognostic aids but need further prospective research.

On the other hand, various arguments have been made against the multibiomarker approach. Feistritzer et $a l^{98}$ found that the predictive value of hs-cTnT was not further improved when combined with CK, hs-CRP, LDH, AST and ALT. Others have demonstrated that once a single strong predictor of risk (eg, cTn) is included in a combination of markers, it is difficult to quantify the contribution of the latter to predictive models. The relative risk relationships for individual biomarkers and specific end points differ, which can introduce variability in the risk assessment and influence therapeutic decision-making.

\section{SUMMARY}

In this review, we summarise the biomarkers discovered in recent years and focus on their diagnostic and prognostic value in patients with AMI (table 1). The ideal biomarkers of AMI should be sensitive and specific in the early period after the onset of symptoms and provide prognostic information for risk assessment, which could guide clinicians to identify the best treatment options. cTn is a highly sensitive and specific cardiac biomarker for AMI diagnosis and can independently predict adverse cardiac events, including death and heart failure. Other biomarkers such as BNP/NT-proBNP and CRP are also valuable predictors of cardiovascular prognosis. In recent years, several novel biomarkers have been identified for the diagnosis and risk assessment in patients with AMI. A multibiomarker approach can potentially enhance the diagnostic and prognostic accuracy and may have broad clinical application prospects.
Contributors $Y C, Y T$ and $L Z$ wrote the manuscript. WX and $X Z$ revised the manuscript.

Funding This study was financially supported by the National Natural Science Foundation of China (no. 81770370) and Scientific Research Program for Young Talents of China National Nuclear Corporation (no. 51001).

Competing interests None declared.

Patient consent for publication Not required.

Provenance and peer review Not commissioned; externally peer reviewed.

\section{REFERENCES}

1. KarmeN A, Wroblewski F, Ladue JS. Transaminase activity in human blood. J Clin Invest 1955;34:126-33.

2. Dolci A, Panteghini M. The exciting story of cardiac biomarkers: from retrospective detection to gold diagnostic standard for acute myocardial infarction and more. Clin Chim Acta 2006;369:179-87.

3. Ladenson JH. A personal history of markers of myocyte injury [myocardial infarction]. Clin Chim Acta 2007;381:3-8.

4. Nowakowski JF. Use of cardiac enyzmes in the evaluation of acute chest pain. Ann Emerg Med 1986:15:354-60.

5. Troponin T and myocardial damage. Lancet 1991;338:23-4.

6. Katus HA, Remppis A, Looser $\mathrm{S}$, et al. Enzyme linked immuno assay of cardiac troponin T for the detection of acute myocardial infarction in patients. J Mol Cell Cardiol 1989:21:1349-53.

7 Myocardial infarction redefined - a consensus document of the joint European Society of Cardiology/American College of cardiology Committee for the redefinition of myocardial infarction. Eur Heart J 2000;21:1502-13.

8. Sacks DB. Acute coronary ischemia: troponin I and T. Vasc Med 1999;4:253-6.

9. Morrow DA, Cannon CP, Jesse RL, et al. National Academy of Clinical Biochemistry Laboratory Medicine Practice Guidelines: clinical characteristics and utilization of biochemical markers in acute coronary syndromes. Clin Chem 2007:53:552-74.

10. Apple FS, Collinson PO, for the IFCC Task Force on Clinical Applications of Cardiac Biomarkers. Analytical characteristics of high-sensitivity cardiac troponin assays. Clin Chem 2012;58:54-61.

11. Chenevier-Gobeaux C, Bonnefoy-Cudraz Éric, Charpentier S, et al. High-sensitivity cardiac troponin assays: answers to frequently asked questions. Arch Cardiovasc Dis 2015;108:132-49.

12. Conrad MJ, Jarolim P. Cardiac troponins and high-sensitivity cardiac troponin assays. Clin Lab Med 2014;34:59-73.

13. Body R. Acute coronary syndromes diagnosis, version 2.0: tomorrow's approach to diagnosing acute coronary syndromes? Turk J Emerg Med 2018;18:94-9.

14. Manikkan AT. Elevated troponin I levels in diabetic ketoacidosis without obstructive coronary artery disease. J Endocr Soc 2018;2:1020-3.

15. Stacy SR, Suarez-Cuervo C, Berger Z, et al. Role of troponin in patients with chronic kidney disease and suspected acute coronary syndrome. Ann Intern Med 2014;161:502-12.

16. Gupta S, Alagona P. Troponins: not always a myocardial infarction. Am J Med 2008;121.

17. Mueller M, Vafaie M, Biener $M$, et al. Cardiac troponin T: from diagnosis of myocardial infarction to cardiovascular risk prediction. Circ J 2013;77:1653-61.

18. Das B, Mishra TK, Trinath KM. Cardiac troponins: current status. JICC 2017:7:1-5

19. Tan NS, Goodman SG, Cantor WJ, et al. Efficacy of early invasive management after fibrinolysis for ST-segment elevation myocardial infarction in relation to initial troponin status. Can J Cardiol 2016;32:1221.e11-1221.e18.

20. Rebalka IA, Hawke TJ. Potential biomarkers of skeletal muscle damage. Biomark Med 2014:8:375-8

21. Zager RA. Marked protection against acute renal and hepatic injury after nitrited myoglobin + tin protoporphyrin administration. Trans/ Res 2015;166:485-501.

22. Newby LK, Storrow AB, Gibler WB, et al. Bedside multimarker testing for risk stratification in chest pain units: the chest Pain evaluation by creatine kinase-MB, myoglobin, and troponin I (CHECKMATE) study. Circulation 2001;103:1832-7.

23. McCord J, Nowak RM, Hudson MP, et al. The prognostic significance of serial myoglobin, troponin I, and creatine kinase-MB measurements in patients evaluated in the emergency department for acute coronary syndrome. Ann Emerg Med 2003;42:343-50.

24. Kontos MC, Garg R, Anderson FP, et al. Ability of myoglobin to predict mortality in patients admitted for exclusion of myocardial infarction. Am J Emerg Med 2007;25:873-9.

25. Mehta M, Marwah S, Ghosh S, et al. A synergistic role of ischemia modified albumin and high-sensitivity troponin $\mathrm{T}$ in the early diagnosis of acute coronary syndrome. J Family Med Prim Care 2015:4:570-5.

26. Manini AF, Ilgen J, Noble VE, et al. Derivation and validation of a sensitive IMA cutpoint to predict cardiac events in patients with chest pain. Emerg Med J 2009;26:791-6.

27. Kim Y, Kim H, Kim S-Y, et al. Automated heart-type fatty acid-binding protein assay for the early diagnosis of acute myocardial infarction. Am J Clin Pathol 2010;134:157-62. 
28. Agnello L, Bivona G, Novo G, et al. Heart-type fatty acid binding protein is a sensitive biomarker for early AMI detection in troponin negative patients: a pilot study. Scand J Clin Lab Invest 2017;77:428-32.

29. Reiter M, Twerenbold R, Reichlin T, et al. Heart-type fatty acid-binding protein in the early diagnosis of acute myocardial infarction. Heart 2013;99:708-14.

30. Erlikh AD, Katrukha AG, Trifonov IR, et al. Prognostic significance of heart fatty acid binding protein in patients with non-ST elevation acute coronary syndrome:results of follow up for 12 months. Kardiologia 2005;45:13-21.

31. Jones JD, Chew PG, Dobson R, et al. The prognostic value of heart type fatty acid binding protein in patients with suspected acute coronary syndrome: a systematic review. Curr Cardiol Rev 2017;13:189-98.

32. XD Y, He Y, Wang S, et al. Heart-type fatty acid binding protein (H-FABP) as a biomarker for acute myocardial injury and long-term post-ischemic prognosis. Acta Pharmacol Sin 2018;39:1155-63.

33. Richards AM, Nicholls MG, Espiner EA, et al. B-type natriuretic peptides and ejection fraction for prognosis after myocardial infarction. Circulation 2003;107:2786-92.

34. Staub D, Nusbaumer C, Zellweger MJ, et al. Use of B-type natriuretic peptide in the detection of myocardial ischemia. Am Heart J 2006;151:1223-30.

35. Niu JM, Ma ZL, Xie C, et al. Association of plasma B-type natriuretic peptide concentration with myocardial infarct size in patients with acute myocardial infarction. Genet Mol Res 2014;13:6177-83.

36. Park M, Vittinghoff E, Shlipak MG, et al. Associations of N-terminal pro-B-type natriuretic peptide with kidney function decline in persons without clinical heart failure in the Heart and Soul Study. Am Heart J 2014;168:931-9.

37. Drewniak W, Szybka W, Bielecki D, et al. Prognostic significance of NT-proBNP levels in patients over 65 presenting acute myocardial infarction treated Invasively or conservatively. Biomed Res Int 2015;2015:1-6.

38. Islam MN, Alam MF, Debnath RC, et al. Correlation between troponin-I and B-type natriuretic peptide level in acute myocardial infarction patients with heart failure. Mymensingh Med J 2016;25:226-31.

39. Reesukumal K. Pratumvinit B. B-type natriuretic peptide not TIMI risk score predicts death after acute coronary syndrome. Clin Lab 2012;58:1017-22.

40. Khan SQ, Quinn P, Davies JE, et al. N-terminal pro-B-type natriuretic peptide is better than TIMI risk score at predicting death after acute myocardial infarction. Heart 2008;94:40-3.

41. Hamid SA, Baxter GF. Adrenomedullin: regulator of systemic and cardiac homeostasis in acute myocardial infarction. Pharmacol Ther 2005; 105:95-112.

42. Yuyun MF, Narayan HK, Ng LL. Prognostic significance of adrenomedullin in patients with heart failure and with myocardial infarction. Am J Cardiol 2015;115:986-91.

43. Liu J, Masoudi FA, Spertus JA, et al. Patterns of use of angiotensin-converting enzyme Inhibitors/angiotensin receptor blockers among patients with acute myocardial infarction in China from 2001 to 2011: China PEACE-Retrospective AMl study. J Am Heart Assoc 2015;4.

44. Pitt B, Bakris G, Ruilope LM, et al. Serum potassium and clinical outcomes in the Eplerenone Post-Acute Myocardial Infarction Heart Failure Efficacy and Survival Study (EPHESUS). Circulation 2008;118:1643-50.

45. Bonaca MP, Morrow DA, Braunwald E, et al. Growth differentiation factor- 15 and risk of recurrent events in patients stabilized after acute coronary syndrome. Arterioscler Thromb Vasc Biol 2011;31:203-10.

46. Lukin A, Novak K, Polić S, et al. Prognostic value of low and moderately elevated C-reactive protein in acute coronary syndrome: a 2-year follow-up study. Med Sci Monit 2013;19:777-86.

47. Wang J, Tang B, Liu X, et al. Increased monomeric CRP levels in acute myocardial infarction: a possible new and specific biomarker for diagnosis and severity assessment of disease. Atherosclerosis 2015;239:343-9.

48. Correia LC, Vasconcelos I, Garcia G, et al. Does C-reactive protein add prognostic value to GRACE score in acute coronary syndromes? Arq Bras Cardiol 2014;102:449-55.

49. Forte L, Cimmino $G$, Loffredo F, et al. C-reactive protein is released in the coronary circulation and causes endothelial dysfunction in patients with acute coronary syndromes. Int J Cardiol 2011;152:7-12.

50. LP H, Tang XY, Ling WH, et al. Early C-reactive protein in the prediction of long-term outcomes after acute coronary syndromes: a meta-analysis of longitudinal studies. Heart 2010;96:339-46.

51. HamzicMehmedbasic A. Inflammatory cytokines as risk factors for mortality after acute cardiac events. Med Arch 2016;70:252-5.

52. Yousuf $\mathrm{O}$, Mohanty BD, Martin SS, et al. High-sensitivity C-reactive protein and cardiovascular disease: a resolute belief or an elusive link? J Am Coll Cardiol 2013:62:397-408

53. Puri R, Nissen SE, Shao M, et al. Impact of baseline lipoprotein and C-reactive protein levels on coronary atheroma regression following high-intensity statin therapy. Am J Cardiol 2014;114:1465-72.

54. Mueller C. Biomarkers and acute coronary syndromes: an update. Eur Heart $J$ 2014:35:552-6

55. Wang XH, Liu SQ, Wang YL, et al. Correlation of serum high-sensitivity C-reactive protein and interleukin-6 in patients with acute coronary syndrome. Genet Mol Res 2014;13:4260-6.
56. García-Salas JM, Tello-Montoliu A, Manzano-Fernández S, et al. Interleukin-6 as a predictor of cardiovascular events in troponin-negative non-ST elevation acute coronary syndrome patients. Int J Clin Pract 2014;68:294-303.

58 Kubková L, Spinar J, Pávková Goldbergová M, et al. Inflammatory response and $\mathrm{C}$-reactive protein value in patient with acute coronary syndrome. Vnitr Lek 2013;59:981-8.

58. Cherneva ZV, Denchev SV, Gospodinova MV, et al. Inflammatory cytokines at admission-independent prognostic markers in patients with acute coronary syndrome and hyperglycaemia. Acute Card Care 2012;14:13-19.

59. Kafkas N, Venetsanou K, Patsilinakos $\mathrm{S}$, et al. Procalcitonin in acute myocardial infarction. Acute Card Care 2008;10:30-6.

60. Ataoğlu HE, Yilmaz F, Uzunhasan I, et al. Procalcitonin: a novel cardiac marker with prognostic value in acute coronary syndrome. J Int Med Res 2010;38:52-61.

61. Kelly D, Khan SQ, Dhillon O, et al. Procalcitonin as a prognostic marker in patients with acute myocardial infarction. Biomarkers 2010;15:325-31.

62. Anatoliotakis N, Deftereos S, Bouras G, et al. Myeloperoxidase: expressing inflammation and oxidative stress in cardiovascular disease. Curr Top Med Chem 2013;13:115-38

63. Hochholzer W, Morrow DA, Giugliano RP. Novel biomarkers in cardiovascular disease: update 2010. Am Heart J 2010;160:583-94.

64. Omran MM, Zahran FM, Kadry M, et al. Role of myeloperoxidase in early diagnosis of acute myocardial infarction in patients admitted with chest pain. J Immunoassay Immunochem 2018;39:337-47.

65. Mittal B, Mishra A, Srivastava A, et al. Matrix metalloproteinases in coronary artery disease. Adv Clin Chem 2014;64:1-72.

66. Halade GV, Jin Y-F, Lindsey ML. Matrix metalloproteinase (MMP)-9: a proximal biomarker for cardiac remodeling and a distal biomarker for inflammation. Pharmacol Ther 2013;139:32-40.

67. Varo N, de Lemos JA, Libby P, et al. Soluble CD40L. Risk prediction after acute coronary syndromes. Circulation 2003;108:1049-52.

68. Bui $A H$, Bonaca MP, Sabatine MS, et al. Elevated concentration of placental growth factor (PIGF) and long term risk in patients with acute coronary syndrome in the PROVE IT-TIMI 22 trial. J Thromb Thrombolysis 2012;34:222-8.

69. Sun T, Dong Y-H, Du W, et al. The role of microRNAs in myocardial infarction: from molecular mechanism to clinical application. Int J Mo/ Sci 2017:18.

70. Gidlöf O, Smith JG, Miyazu K, et al. Circulating cardio-enriched microRNAs are associated with long-term prognosis following myocardial infarction. BMC CardiovasC Disord 2013;13.

71. Devaux $Y$, Mueller $M$, Haaf $P$, et al. Diagnostic and prognostic value of circulating microRNAs in patients with acute chest pain. J Intern Med 2015;277:260-71.

72. Zhu J, Yao K, Wang Q, et al. Circulating miR-181a as a potential novel biomarker for diagnosis of acute myocardial infarction. Cell Physiol Biochem 2016;40:1591-602.

73. Zhang Y, Cheng J, Chen F, et al. Circulating endothelial microparticles and miR-92a in acute myocardial infarction. Biosci Rep 2017;37.

74. Oerlemans MIFJ, Mosterd A, Dekker MS, et al. Early assessment of acute coronary syndromes in the emergency department: the potential diagnostic value of circulating microRNAs. EMBO Mol Med 2012:4:1176-85.

75. Goretti E, Wagner DR, Devaux Y. miRNAs as biomarkers of myocardial infarction: a step forward towards personalized medicine? Trends Mol Med 2014;20:716-25.

76. Schulte C, Molz S, Appelbaum S, et al. miRNA-197 and miRNA-223 predict cardiovascular death in a cohort of patients with symptomatic coronary artery disease. PLoS One 2015:10:e0145930.

77. He F, Lv P, Zhao X, et al. Predictive value of circulating miR-328 and miR-134 for acute myocardial infarction. Mol Cell Biochem 2014;394:137-44.

78. Lv P, Zhou M, He J, et al. Circulating miR-208b and miR-34a are associated with left ventricular remodeling after acute myocardial infarction. Int J Mo/ Sci 2014; 15:5774-88

79. Kohli P, Bonaca MP, Kakkar R, et al. Role of ST2 in non-ST-elevation acute coronary syndrome in the MERLIN-TIMI 36 trial. Clin Chem 2012;58:257-66.

80. Salvagno GL, Pavan C. Prognostic biomarkers in acute coronary syndrome. Ann Trans/ Med 2016;4

81. Zhang K, Zhang XC, YH M, et al. Predicting value of serum soluble ST2 and interleukin-33 for risk stratification and prognosis in patients with acute myocardial infarction. Chin Med J 2013:126:3628-31.

82. Adela R, Banerjee SK. GDF-15 as a target and biomarker for diabetes and cardiovascular diseases: a translational prospective. J Diabetes Res 2015;2015:1-14.

83. Schaub N, Reichlin T, Twerenbold R, et al. Growth differentiation factor-15 in the early diagnosis and risk stratification of patients with acute chest pain. Clin Chem 2012;58:441-9.

84. Kempf T, Bjorklund E, Olofsson S, et al. Growth-differentiation factor-15 improves risk stratification in ST-segment elevation myocardial infarction. Eur Heart $J$ 2007;28:2858-65

85. Kang Q, Li X, Yang M, et al. Galectin-3 in patients with coronary heart disease and atrial fibrillation. Clin Chim Acta 2018;478:166-70

86. Bivona G, Bellia C, Lo Sasso B, et al. Short-term changes in gal 3 circulating levels after acute myocardial infarction. Arch Med Res 2016;47:521-5. 
87. González GE, Cassaglia P, Noli Truant S, et al. Galectin-3 is essential for early wound healing and ventricular remodeling after myocardial infarction in mice. Int I Cardiol 2014; 176:1423-5.

88. Lisowska A, Knapp M, Tycińska A, et al. Predictive value of galectin-3 for the occurrence of coronary artery disease and prognosis after myocardial infarction and its association with carotid IMT values in these patients: a mid-term prospective cohort study. Atherosclerosis 2016;246:309-17.

89. Wang A, Zhong C, Zhu Z, et al. Serum galectin-3 and poor outcomes among patients with acute ischemic stroke. Stroke 2018;49:211-4.

90 David del VM, Marcelo SF, Jose Luís ZG. Biomarkers in acute coronary syndrome. IJC Metabolic \& Endocrine 2015;8:20-3.

91. Kim H, Yang DH, Park Y, et al. Incremental prognostic value of C-reactive protein and $\mathrm{N}$-terminal ProB-type natriuretic peptide in acute coronary syndrome. Circ J 2006;70:1379-84.

92. O'Donoghue ML, Morrow DA, Cannon CP, et al. Multimarker risk stratification in patients with acute myocardial infarction. J Am Heart Assoc 2016;5.

93. Aldous SJ. Cardiac biomarkers in acute myocardial infarction. Int I Cardiol 2013;164:282-94.

94. Young JM, Pickering JW, George PM, et al. Heart fatty acid binding protein and cardiac troponin: development of an optimal rule-out strategy for acute myocardial infarction. BMC Emerg Med 2016;16.

95. McCann CJ, Glover BM, Menown IBA, et al. Prognostic value of a multimarker approach for patients presenting to hospital with acute chest pain. Am J Cardiol 2009:103:22-8
96. Oemrawsingh RM, Lenderink T, Akkerhuis KM, et al. Multimarker risk model containing troponin-T, interleukin 10, myeloperoxidase and placental growth factor predicts long-term cardiovascular risk after non-ST-segment elevation acute coronary syndrome. Heart 2011;97:1061-6.

97. Schernthaner $\mathrm{C}$, Lichtenauer $\mathrm{M}$, Wernly $\mathrm{B}$, et al. Multibiomarker analysis in patients with acute myocardial infarction. Eur J Clin Invest 2017;47:638-48.

98. Feistritzer $\mathrm{H}-\mathrm{J}$, Reinstadler SJ, Klug G, et al. Multimarker approach for the prediction of microvascular obstruction after acute ST-segment elevation myocardial infarction: a prospective, observational study. BMC Cardiovasc Disord 2016;16.

\section{Answers}

1. A-True; B-True; C-False; D-True

2. A-True; B-True; C-True; D-True

3. A-False; B-False; C-False; D-True

4. A-True; B-True; C-True; D-True

5. A-True; B-False; C-True; D-False 